\title{
The First Described Case of Bizarre Leiomyoma of the Portal Vein
}

\author{
Murat Ferhat Ferhatoglu \\ Department of General Surgery, Faculty of Medicine, Okan University, Istanbul, Turkey
}

\begin{abstract}
Vascular leiomyomas with bizarre nuclei are rare. Differentiation of these tumors from malignant lesions is essential to avoid unnecessary dissection and organ loss in surgery. Herein, we present a 39-year Kosovo woman with a bizarre leiomyoma arising from the portal vein. The patient presented to the general surgery clinic with a one-year history of abdominal pain, vomiting and back ache. No abnormality was observed on physical examination. On computed tomography, a $42 \times 35 \mathrm{~mm}$, cystic-solid mass in the pancreatic neck, wrapping the superior mesenteric vein for 360-degrees and the superior mesenteric artery for 180-degrees, was found.

In contrast to preoperative findings, a $5 \times 4 \mathrm{~cm}$ mass located behind the portal vein and reaching portal vein-superior mesenteric vein junction posteriorly was seen perioperatively. There was no relationship between the mass and the superior mesenteric artery. The tumor was excised with the posterior wall of the junction of the portal vein and the superior mesenteric vein. The end-to-end vascular anastomosis was performed between the portal vein and the superior mesenteric vein. After finishing distal pancreatectomy, the operation was accomplished without any complication. The patient was discharged on 10th day of the operation without any problem.

In conclusion, vascular bizarre leiomyomas are rare tumors. It is impossible to diagnose these correctly before surgery. Perioperative use of pathological examination as a frozen section can be helpful to avoid unnecessary surgical procedures.
\end{abstract}

Key Words: Bizarre leiomyoma, leiomyosarcoma, portal vein.

How to cite this article: Ferhatoglu MF. The first described case of bizarre leiomyoma of the portal vein. $J$ Coll Physicians Surg Pak 2019; 29 (Supplement 2):S123-S125.

\section{INTRODUCTION}

Bizarre leiomyoma (BLM), a rare type of smooth muscle tumor, is a benign tumor that arises from smooth muscle cells, and its histological features include atypical, pleomorphic, bizarre nuclei with or without few mitoses and karyorrhectic nuclei that may mimic atypical mitoses.1-5 Although histologically similar to leiomyosarcoma, the biological behaviour of BLM does not differ from that of conventional leiomyoma (LM). To avoid unnecessary treatment, a differential diagnosis should be made to distinguish BLM from leiomyosarcoma by strict criteria (LMS). ${ }^{6}$

To the best of my knowledge, there are no published cases of BLM of the portal vein in the literature. In the present case, I report the clinical manifestations and the imaging and pathological findings of the first case of BLM arising from the portal vein in a 39-year female patient. Written consent was obtained from the patient.

\section{CASE REPORT}

A 39-year female of Kosovo ethnicity presented to the general surgery clinic with a 1-year history of abdominal

Correspondence to: Murat Ferhat Ferhatoglu, Aydinli Yolu

Caddesi, Okan Üniversitesi Hastanesi, Tuzla, Istanbul, Turkey

E-mail: ferhatferhatoglu@yahoo.co.uk

Received: February 04, 2019; Revised: April 26, 2019;

Accepted: May 03, 2019 pain, vomiting, and back ache. Physical examination and blood tests revealed no abnormalities. A 4-cm mass in the pancreatic head was observed by abdominal ultrasonography. Computed tomography (CT) revealed a $42 \times 35 \mathrm{~mm}$ solid mass in the pancreatic neck, wrapping the superior mesenteric vein by $360^{\circ}$ and the superior mesenteric artery by $180^{\circ}$ (Figure 1 ). The patient was diagnosed as a pancreatic mass, suspected to be malignant; and a tru-cut needle biopsy was planned to target the mass. Following the biopsy, a diagnosis of smooth muscle tumor was reported in the pathology results, and the patient was prepared for surgery. Preoperatively, the use of a saphenous vein graft was planned for the reconstruction of infiltrated vessels.

In contrast to the preoperative diagnosis, a $5 \times 4 \mathrm{~cm}$ mass located behind the portal vein and infiltrating into the portal vein-superior mesenteric vein junction posteriorly was observed intraoperatively (Figure 2). There was no relationship between the mass and the trunk of the superior mesenteric artery. The tumor was excised from the posterior wall of the junction of the portal vein with the superior mesenteric vein. To avoid unnecessary dissection, frozen sections were sent, and the mass was diagnosed in compliance with previous tru-cut biopsies as a low-grade smooth muscle tumor. The end-to-end vascular anastomosis was performed between the portal vein and the superior mesenteric vein without any complications. 
A macroscopic examination of the specimen showed a fibrous, solid, homogeneous, appearance. On crosssection, it showed a nodular, well-defined, gray-white color tumor with dimensions of $5.5 \times 4.5 \times 3.5 \mathrm{~cm}$. Microscopic examination revealed a tumor composed of

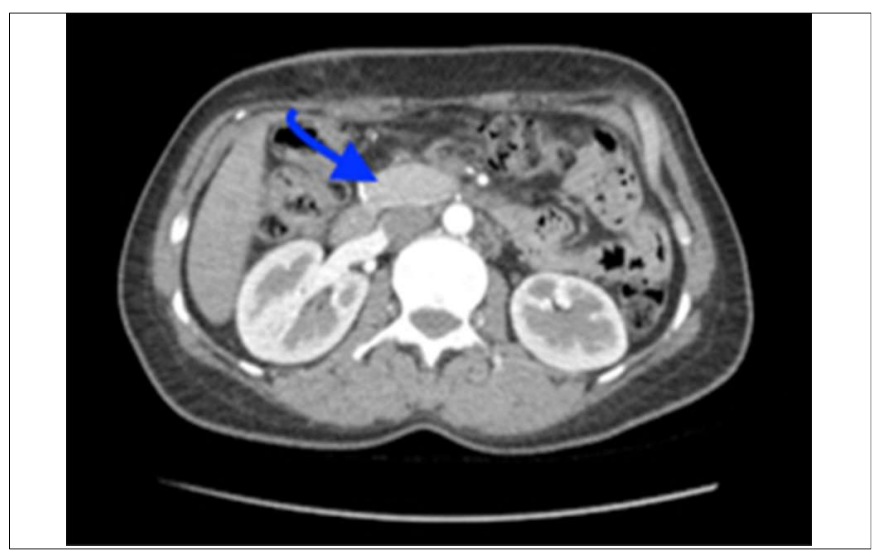

Figure 1: Cystic-solid mass infiltrating inferior mesenteric vein (Blue arrow).

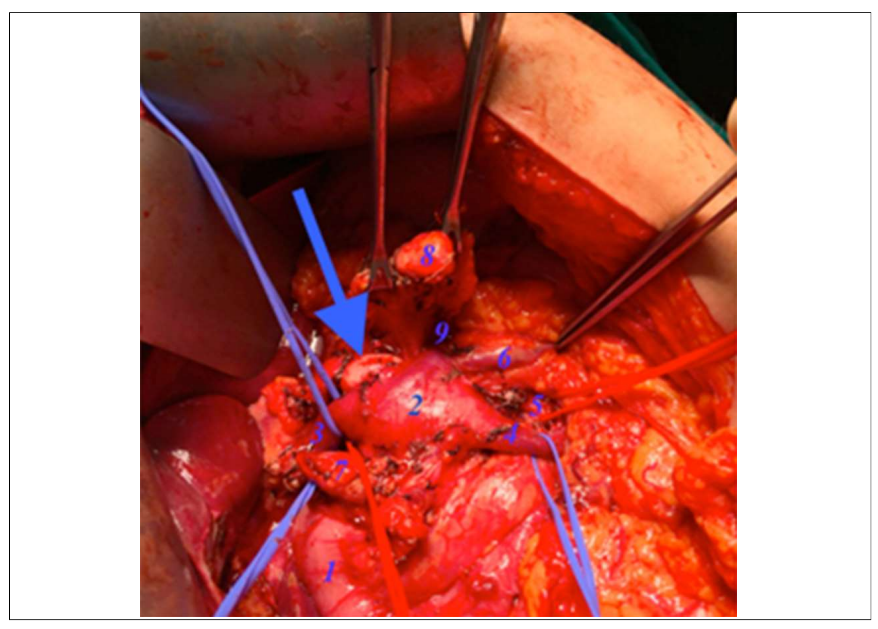

Figure 2: Perioperative view of the lesion (Blue arrow), 1: duodenum, 2: conjunction of the portal and the superior mesenteric veins, 3: portal vein, 4: superior mesenteric vein, 5 : superior mesenteric artery, 6 : inferior mesenteric vein, $7:$ neck of pancreas, 8 : body of the pancreas, 9 : splenic vein.

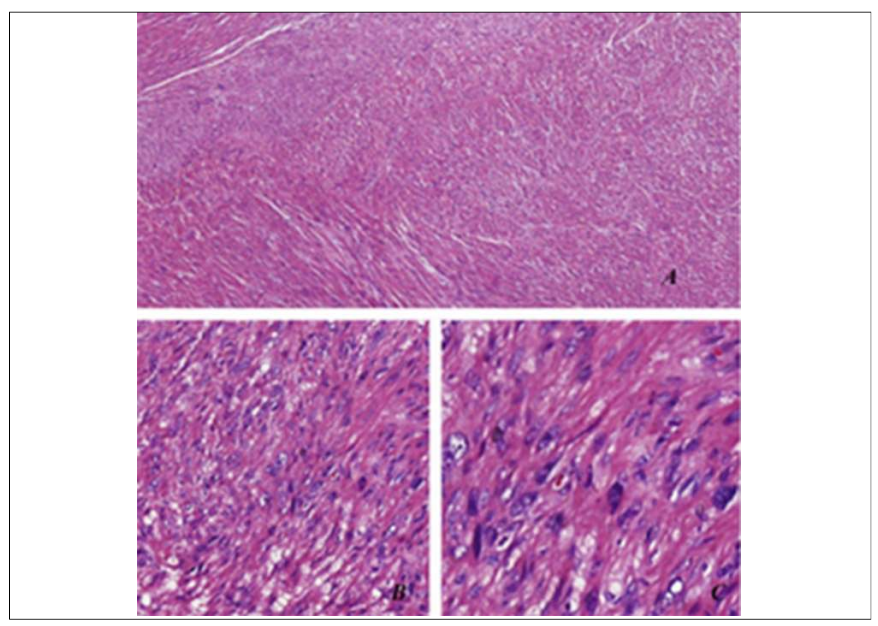

Figure 3: Bizarre leiomyoma hematoxylin section: (A) 10X HPF, (B) 20X HPF, (C) 40X HPF. fusiform cells (Figure 3). Tumor cells contained bizarre nuclei; and 4 mitoses were seen within the tumor in 50 high power field (HPF). Atypical mitoses and necrosis were not observed. Immunohistochemically, the tumor stained positive for desmin, SMA, and caldesmon and negative for CD34, S-100, CD-117, and PAN-CK (Figure 4). Furthermore, Ki-67 labelling was less than $5 \%$. In the light of these morphological and immunohistochemical findings, the case was diagnosed as BLM.

The patient stayed in the hospital for 10 days, including one day in the intensive care unit. Her drains were removed on the $7^{\text {th }}$ postoperative day, and she was discharged uneventfully on the 10th day. No problem was found on routine following of patient at 1 st and 3rd postoperative months.

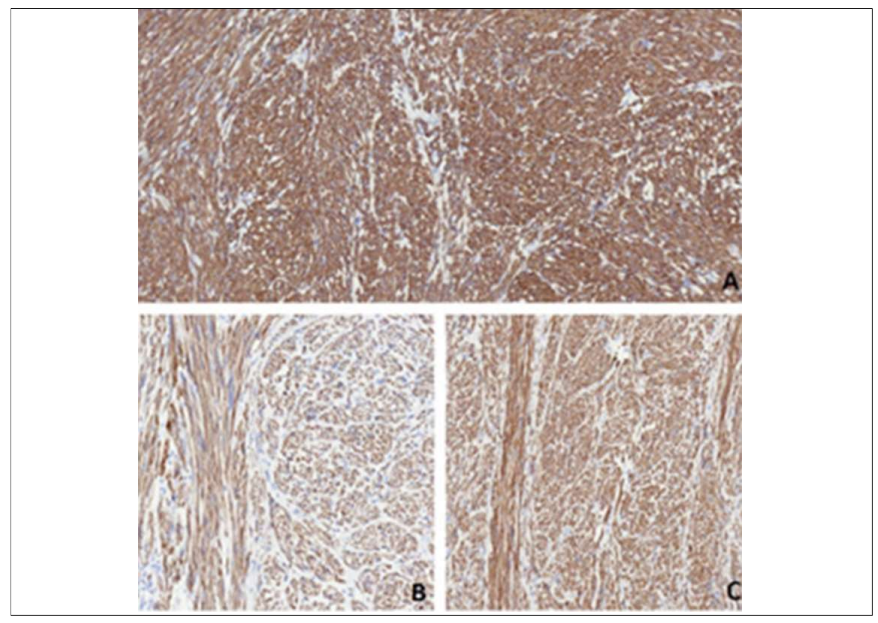

Figure 4: Bizarre leiomyoma immunohistochemistry: (A) SMA, (B) Caldesmon, (C) Desmin 20X HPF.

\section{DISCUSSION}

Primary smooth muscle tumors originating from great vessel walls are quite rare; pulmonary veins are the most frequently affected site, followed by the aorta and inferior vena cava. ${ }^{6}$ A previous study reports only 115 vessel LM cases, one of which describes LM of the portal vein. ${ }^{7}$ However, the present case is unique in that it describes BLM arising from the wall of the portal vein. Two types of peripheral soft tissue LMs have been described. Gynecologic LMs are more common and typically affect women; whereas, somatic LMs are relatively rare and usually occur in the deep somatic soft tissue of the extremities. Somatic LMs have been the focus of case reports in the literature. ${ }^{8}$ Another unique type of these tumors are BLMs, which were first described by Kelly and Cullen in 1909; before this, they were named by different authors as "degenerative cytological change", "symplastic cells" and "leiomyosarcoma in situ". In 1972, Christopherson first used the word "bizarre" in the description of this lesion. Although frequently confused, the absence of tumor cell necrosis and a high mitotic rate serve to differentiate BLMs from LMs. ${ }^{9}$ The requirements for diagnosis of BLMs are as 
follows: 1) unquestionable smooth muscle cell type; 2) the presence of pleomorphic bizarre multinucleated tumor giant cells, comprising a minimum of $5 \%$ of the tumor; and 3) a mitoses count of 10/10 HPF in the most mitotically active areas of the tumor. ${ }^{10}$ Immunohistochemical studies with a panel of antibodies against p16, p53, and Ki-67 help distinguish LMs from both cellular and usual LMs. However, due to significant overlapping of the staining patterns between LMs and BLMs, immunostaining for $\mathrm{p} 16, \mathrm{p} 53$, and $\mathrm{Ki}-67$ play a limited role in differentiating LMs from BLMs. ${ }^{11}$

BLMa are known to be clinically benign. The absolute criteria of malignancy are high mitotic index, nuclear atypia, and necrosis. ${ }^{12}$ Mitotic activity in BLM is low compared with that in LMs. In the study by Downes and Hart, involving 24 BLMs, 0-7 mitoses were found per 50-HPF. 13

LMs occur most frequently in the genitourinary system and gastrointestinal system. Clinically, these neoplasms may have varying sizes, symptomless, or have symptoms according to its localisation. In our case, the patient had diffuse abdominal and back ache caused by pressure and semi-obstruction of the junction of the portal vein. 8

Preoperative diagnosis of BLMs is difficult, and these tumors are frequently misdiagnosed as perivascular or malignant lesions. In our case, the radiological examination misdiagnosed the tumor as a cystic, solid tumor of the pancreas head.

Magnetic resonance imaging is more useful than CT in defining the characteristics of this type of vascular tumor; this is due to the fibrous stroma and smooth muscle that form the lesion. In the present case, only CT was performed, resulting in the misdiagnosis of the tumor as a pancreatic head mass. ${ }^{14}$

Surgical resection of the tumor is the best treatment choice for vascular smooth muscle tumors ${ }^{15}$, and en-bloc resection with vessels should be performed, if necessary. En-bloc resection of the mass with the vessel wall was performed in my patient. Analysis of the frozen sections confirmed that the lesion was not malignant, and the operation was terminated with limited instead of aggressive surgery.

In conclusion, venous BLMs are rare tumors and are impossible to diagnose preoperatively. Histopathological examination of the excised tumor is the only approach to establish an exact diagnosis. Perioperative use of pathological examination using frozen sections can help avoid unnecessary surgical procedures.

\section{PATIENT'S CONSENT:}

Written consent was obtained from the patient.

\section{CONFLICT OF INTEREST:}

Authors declared no conflict of interest.

\section{AUTHOR'S CONTRIBUTION:}

MFF: Collected the information, reviewed the literature and wrote the manuscript.

\section{REFERENCES}

1. Farman AG. Benign smooth muscle tumours. $S$ Afr Med $J$ 1975; 49:1333-40.

2. Turashvili G, Manduch M, Varma S. Somatic soft tissue leiomyoma in an elderly patient: $A$ case report and review of existing literature. Int J Pathol 2015; 13:32-5.

3. Billings SD, Folpe AL, Weiss SW. Do leiomyomas of deep soft tissue exist? An analysis of highly differentiated smooth muscle tumors of deep soft tissue supporting two distinct subtypes. Am J Surg Pathol 2001; 25:1134-42.

4. McCarthy AJ, Chetty R. Benign smooth muscle tumors (Leiomyomas) of deep somatic soft tissue. Sarcoma 2018; 2018:2071394.

5. Croce $\mathrm{S}$, Young $\mathrm{RH}$, Oliva E. Uterine leiomyomas with bizarre nuclei: A clinicopathologic study of 59 cases. Am J Surg Pathol 2014; 38:1330-9.

6. Chetty R, Kalimuthu SN, Heinonen HR. Primary inferior vena cava smooth muscle tumor with diffuse bizarre giant nuclei and low mitotic rate: a nomenclatural conundrum. Cardiovasc Pathol 2017; 30:1-5.

7. Kremer K, Palomba PP, Sandmann W. Leiomyoma of the portal vein. Chirurg 1976; 47:214-20.

8. Hashimoto H, Quade B. Leiomyoma of deep soft tissue. Ed. 1st, Lyon: IARC press; 2002.

9. Kefeli M, Caliskan S, Kurtoglu E. Leiomyoma with bizarre nuclei: Clinical and pathologic features of 30 patients. Int $J$ Gynecol Pathol 2017; 37:379-87.

10. Downes KA, Hart WR. Bizarre leiomyomas of the uterus: A comprehensive pathologic study of 24 cases with long-term follow-up. Am J Surg Pathol 1997; 21:1261-70.

11. Chen L, Yang B. Immunohistochemical analysis of p16, p53 and $\mathrm{Ki}-67$ expression in uterine smooth muscle tumors. Int $\mathrm{J}$ Gynecol Pathol 2008; 27:326-32.

12. Losch A, Haider-Angeler MG, Kainz C, Breitenecker G, Lahodny J. Leiomyoma of the round ligament in a postmenopausal woman. Maturitas 1999; 31:133.

13. Bell SW, Kempson RL, Hendrickson MR. Problematic uterine smooth muscle neoplasms. Am J Surg Pathol 1994; 18: 535.

14. Xu L, Jin X, Han Z. Leiomyoma of the femoral vein: A case report and review of the literature. Ann Vasc Surg 2014; 28:5-9.

15. Liu B, Liu C, Guan H. Intravenous leiomyomatosis with inferior vena cava and hearth extension. J Vasc Surg 2009; 50:897-902. 